\title{
Antimicrobial Susceptibility of Quinupristin/Dalfopristin Tested Against Gram-Positive Cocci From Latin America: Results from the Global SMART (GSMART) Surveillance Study
}

\author{
Helio S. Sader, Ronald N. Jones, Charles H. Ballow, \\ Douglas J. Biedenbach, Rosangela F. Cereda and \\ GSMART Latin America Study Group
}

\author{
Division of Infectious Diseases, Federal University of \\ São Paulo, Brazil; Department of Pathology, \\ University of Iowa College of Medicine, Iowa City, IA, \\ USA; Clinical Pharmacokinetics Laboratory, Millard \\ Fillmore Hospital, Buffalo, NY, USA
}

\begin{abstract}
Gram-positive cocci are important causes of both nosocomial and community-acquired infections, and antimicrobial resistance among these pathogens has become an important problem worldwide. Since resistance among these organisms can vary substantially by geographic location, we conducted a multicenter surveillance study with isolates from five Latin American countries (15 medical centers). Quinupristin/dalfopristin (formerly RP-59500) is a novel streptogramin combination with focused activity against Gram-positive cocci, many exhibiting emerging resistance. The in vitro activity of quinupristin/dalfopristin and 12 other antimicrobial agents were evaluated against 1,948 strains including Staphylococcus aureus (747 strains), coagulase-negative staphylococci (CoNS; 446 strains), enterococci (429 strains), and various Streptococcus spp. (326 strains). Oxacillin resistance was observed in $41 \%$ of $S$. aureus (MIC, $\leq 2 \mu \mathrm{g} / \mathrm{ml}$ or $\geq 13 \mathrm{~mm}$ ) and $40 \%$ of CoNS (MIC, $\leq 0.25 \mu \mathrm{g} / \mathrm{ml}$ or $\geq 18 \mathrm{~mm}$ ). Vancomycin, teicoplanin, and quinupristin/dalfopristin $\left(\mathrm{MIC}_{90}, 0.25-1 \mu \mathrm{g} / \mathrm{ml}\right)$ remained effective against all strains, but cross-resistance was high among other tested drugs. The quinupristin/ dalfopristin $\mathrm{MIC}_{50}$ for Streptococcus pneumoniae and other streptococci was only $0.5 \mu \mathrm{g} / \mathrm{ml}(13 \%$ to $28 \%$ were penicillin-resistant; $12 \%$ to $22 \%$ were macrolide-resistant). Enterococci demonstrated variable inhibition by quinupristin/dalfopristin depending upon identification and the susceptibility testing method used. The demonstrated quinupristin/dalfopristin activity against Enterococcus faecium was confirmed, but potential species identification errors with various commercial systems continue to confuse susceptibility statistics, even though some strains of $E$. faecium confirmed by PCR-based or other molecular identification techniques did have quinupristin/dalfopristin MICs of $\geq 4 \mu \mathrm{g} / \mathrm{mL}$. Most important, glycopeptide-resistant enterococci are rapidly emerging in Latin America, and quinupristin/ dalfopristin appears active against many of these isolates as well as having potency against nearly all staphylococci and streptococci tested at $\leq 2 \mu \mathrm{g} / \mathrm{ml}$ or having a zone diameter of $\geq 16 \mathrm{~mm}$. Comparisons to GSMART results from other continents show nearly identical quinupristin/dalfopristin activity for each Gram-positive species tested. These results define the role of quinupristin/dalfopristin in Latin American medical centers and provide a benchmark for future in vitro comparisons.

Key Words: Quinupristin/dalfopristin, Gram-positive cocci, SMART, antimicrobial surveillance.
\end{abstract}

Received on 30 June 2000; revised 20 October 2000.

Address for correspondence: Helio S. Sader, M.D. Laboratório Especial de Microbiologia Clínica - Infectious Disease Division - Federal University of São Paulo. Rua Botucatu, 740. São Paulo, SP -Zip Code: 04023-063 - Brazil. Phone: + (55 11) 5715180/576-4393/5081-2819. Fax: + (55 11) 543-3013/571-5180. E-mail: heliosader@uol.com.br

The Brazilian Journal of Infectious Diseases 2001;5(1):21-31 (C) 2001 by The Brazilian Journal of Infectious Diseases and Contexto Publishing. All rights reserved.

$1413-8670$
Several studies have shown a progressive increase in antimicrobial resistance among Gram-positive cocci, especially in the United States (U.S.). Vancomycinresistant enterococci (VRE) appeared and disseminated all over the U.S. in the early 1990s. Pfaller and colleagues [1] in the SENTRY Antimicrobial Surveillance Program listed a vancomycin resistance rate in bloodstream enterococci at $16 \%$ to $18 \%$ in 1997, having increased from less than $1 \%$ in 1989. However, in spite of the continuing increase in the 
vancomycin resistance rates among enterococci in the U.S., this pathogen has rarely been described in Latin American countries [1-3].

Oxacillin resistance among staphylococci has been a chronic problem in most U.S. and Latin American tertiary care hospitals. Data from the SENTRY Program has shown that $30 \%$ to $50 \%$ of Staphylococcus aureus strains collected in Latin American hospitals were resistant to oxacillin and most of the anti-staphylococci drugs available, except the glycopeptides [3, 4]. Fortunately, $S$. aureus strains with reduced susceptibility to glycopeptides have not been reported in Latin America yet [5]. However, glycopeptide (teicoplanin) resistance has been reported among coagulase-negative staphylococci (CoNS) in Brazil, especially among $S$. haemolyticus. Using the published susceptible breakpoint for oxacillin $(\geq 0.25$ $\mu \mathrm{g} / \mathrm{mL}$ ), resistance to this antimicrobial agent can reach $75 \%$ among CoNS strains in Latin American medical centers [6].

In the search for therapeutic agents to treat serious Gram-positive infections, the streptogramin combination of quinupristin/dalfopristin (Synercid $\AA$ ) has been widely studied [7]. In this work, we evaluated the in vitro activity of quinupristin/dalfopristin against Gram-positive cocci collected in 15 Latin American medical centers, as part of the international surveillance program called Global Synercid® Microbiologic Assessment of Resistance Trends (GSMART).

\section{Materials and Methods}

Study Design. Fifteen medical centers were recruited in South America to participate in a study known as GSMART. Six of these centers were located in Brazil, 5 in Argentina, 2 in Chile, 1 in Ecuador, and 1 in Venezuela. In Brazil, the medical centers were distributed among 4 cities including São Paulo (3 centers), Rio de Janeiro (1 center), Florianópolis (1 center) and Porto Alegre (1 center). The Argentinean centers were located in Buenos Aires, San Isidro, Cordoba, Rosário and Tandil. Both Chilean centers were in Santiago, and the centers in Ecuador and
Venezuela were located in Quito and Caracas, respectively. The participants performed all in vitro susceptibility tests on-site for approximately 150 strains of Gram-positive organisms using reagents provided.

Organisms. Each participating site was asked to process the following Gram-positive bacterial species (150 total strains): 1) oxacillin-susceptible $S$. aureus (30 strains) and oxacillin-resistant S. aureus (20 strains); 2) oxacillin-susceptible CoNs (15 strains) and oxacillinresistant CoNs (20 strains); 3) Enterococcus faecalis (10 strains) and Enterococcus faecium (30 strains), or 40 strains of enterococci that were not identified to the species level; and 4) 20 strains of Streptococcus pneumoniae and 5 strains of other streptococci which could include viridans gr. and $\beta$-haemolytic streptococci. Various demographic information was collected including body site of infection, hospital unit/ service, and date of culture. All strains were collected from patient infections in 1998, and the first trimester of 1999. Clinical specimens included blood, cerebral spinal fluid, skin and skin structure, bone, intraabdominal infections, and lower and upper respiratory tract samples.

Susceptibility testing methods. Isolates of Streptococcus spp. were tested by using Etest (AB BIODISK, Solna, Sweden). All other organisms were tested by using either broth microdilution (Brazilian centers) or disk diffusion (all other participants). Broth microdilution trays were manufactured by Dade/ Microscan (Sacramento, CA, US). Susceptibility tests were performed following the procedures described by the National Committee for Clinical Laboratory Standards (NCCLS) [8].

Antimicrobial Agents: S. pneumoniae, $\beta$-haemolytic and viridans gr. streptococci were tested against penicillin, cefotaxime, erythromycin, vancomycin, teicoplanin, and quinupristin/dalfopristin. S. aureus and CoNS were tested against erythromycin, clindamycin, cefazolin, cefotaxime, vancomycin, teicoplanin, ciprofloxacin, chloramphenicol, doxycycline and quinupristin/dalfopristin. Ampicillin and gentamicin were 
added for those participants that used commercial broth microdilution trays. Enterococci were tested against ampicillin, vancomycin, teicoplanin, ciprofloxacin, chloramphenicol, doxycycline, and quinupristin/ dalfopristin.

Subcultures of organisms exhibiting a MIC of $>1$ $\mu \mathrm{g} / \mathrm{mL}$ or an inhibition zone diameter of $<19 \mathrm{~mm}$ for quinupristin/dalfopristin were forwarded to the microbiology monitoring sites at the Federal University of São Paulo (São Paulo, Brazil) and the University of Iowa (Iowa City, IA, US) for further characterization. Identification to species level and susceptibility testing were repeated, and the database was corrected when necessary. The susceptibility testing results were recorded as a MIC $(\mu \mathrm{g} / \mathrm{mL})$ or zone diameter $(\mathrm{mm})$ and then interpreted by NCCLS [8, 9] susceptible criteria as follows: for $S$. pneumoniae, quinupristin/ dalfopristin at $\leq 1 \mu \mathrm{g} / \mathrm{mL}$ or $\geq 19 \mathrm{~mm}$, erythromycin at $\leq 0.25 \mu \mathrm{g} / \mathrm{mL}$ or $\geq 23 \mathrm{~mm}$, vancomycin at $\leq 4 \mu \mathrm{g} / \mathrm{mL}$ or $\geq 15 \mathrm{~mm}$ (staphylococci) or $\geq 17 \mathrm{~mm}$ (enterococci), teicoplanin at $\leq 8 \mu \mathrm{g} / \mathrm{mL}$ or $\geq 14 \mathrm{~mm}$, ciprofloxacin at $\leq 1 \mu \mathrm{g} / \mathrm{mL}$ or $\geq 21 \mathrm{~mm}$, oxacillin at $\leq 2 \mu \mathrm{g} / \mathrm{mL}$ or $\geq 13$ $\mathrm{mm}$ (including CoNS) [10], clindamycin at $\leq 0.5 \mu \mathrm{g} /$ $\mathrm{mL}$ or $\geq 21 \mathrm{~mm}$, doxycycline at $\leq 4 \mu \mathrm{g} / \mathrm{mL}$ or $\geq 16$ $\mathrm{mm}$, ampicillin at $\leq 8 \mu \mathrm{g} / \mathrm{mL}$ or $\geq 17 \mathrm{~mm}$, chloramphenicol at $\leq 8 \mu \mathrm{g} / \mathrm{mL}$ or $\geq 18 \mathrm{~mm}$, and all tested cephalosporins (staphylococci only) at $\leq 8 \mu \mathrm{g}$ / $\mathrm{mL}$ or $\geq 18 \mathrm{~mm}$ for cefazolin and cefuroxime, or at $\geq$ $23 \mathrm{~mm}$ for cefotaxime.

Only data sets with acceptable quality control results were analyzed [8]. Quality control monitoring was performed at the Federal University of São Paulo and at the University of Iowa. The quality control organisms were: S. aureus ATCC 25923 (disk diffusion) or ATCC 29213 (Etest and broth microdilution), and $E$. faecalis ATCC 29212.

Pulsed-field gel electrophoresis (PFGE). PFGE of chromosomal DNA was performed on E. faecium strains resistant to vancomycin and/or quinupristin/ dalfopristin, as described by Pfaller, et al. [11]. Restriction digestion of chromosomal DNA was performed with SmaI and the resultant restriction fragments were resolved in a $1 \%$ agarose gel with a
CHEF-DR II system (Bio-Rad Laboratories, Richmond, CA, US). The pulsed time ranged from $5 \mathrm{~s}$ to $30 \mathrm{~s}$ over $23 \mathrm{hs}$ at $13^{\circ} \mathrm{C}$ and $6 \mathrm{~V} / \mathrm{cm}$. PFGE patterns were considered identical if they shared every band, similar if they differed from one another by only 1 to 3 bands, and different if they differed by 4 or more bands.

\section{Results}

Table 1 presents the Etest results (MICs) for quinupristin/dalfopristin and 5 other antimicrobial agents tested against 244 pneumococci and 82 other streptococcal strains. The quinupristin/dalfopristin $\mathrm{MIC}_{90}$ was $1 \mu \mathrm{g} / \mathrm{mL}$ for both groups, and all streptococcal strains tested were considered susceptible (MIC, $\leq 1 \mu \mathrm{g} / \mathrm{mL}$ ). Twenty-eight percent of the $S$. pneumoniae strains were non-susceptible to penicillin (MIC, $>0.06 \mu \mathrm{g} / \mathrm{ml}$ ), while 12 strains $(5 \%)$ harbored high-level resistance ( $\mathrm{MIC}, \geq 2 \mu \mathrm{g} / \mathrm{mL}$ ). Resistance to cefotaxime was detected in $10 \%$ of the $S$. pneumoniae $\left(\mathrm{MIC}_{90}, 0.5 \mu \mathrm{g} / \mathrm{mL}\right)$ strains and $7 \%$ of the other streptococcal species strains, while resistance to erythromycin was much higher $(12 \%$ among pneumoccci and $22 \%$ among other streptococcal species). All streptococcal isolates were susceptible to vancomycin ( $\mathrm{MIC}_{90}, 0.5 \mu \mathrm{g} / \mathrm{mL}$ to $0.75 \mu \mathrm{g} / \mathrm{mL}$ ).

The results of testing $747 \mathrm{~S}$. aureus strains are summarized in Table 2. Two test methods were used and the results are indexed by the oxacillin susceptibility category. Isolates from the Brazilian centers were tested by broth microdilution while isolates from other countries were evaluated by the disk diffusion method. The oxacillin-susceptible $S$. aureus (OSSA) strains were susceptible to most antimicrobial agents evaluated, although some degree of resistance was detected for erythromycin (12\% to $14 \%$ resistance). In contrast, oxacillin-resistant $S$. aureus (ORSA) showed high rates of resistance to most antimicrobial agents evaluated. Only quinupristin/dalfopristin ( $\mathrm{MIC}_{90}, 0.5 \mu \mathrm{g} / \mathrm{mL}$, $100 \%$ susceptibility), vancomycin ( $\mathrm{MIC}_{90}, 1 \mu \mathrm{g} / \mathrm{mL}$, $100 \%$ susceptibility), and teicoplanin $\left(\mathrm{MIC}_{90}, 0.5 \mu \mathrm{g} /\right.$ $\mathrm{mL}, 97 \%$ to $100 \%$ susceptibility) showed reasonable activity against this pathogen (Table 2 ). 
Table 1. Antimicrobial activity of quinupristin-dalfopristin and 5 other compounds tested against 326 streptococci strains isolated in Latin America

\begin{tabular}{|c|c|c|c|c|}
\hline \multirow[t]{2}{*}{ Organisms (no. tested) } & \multirow{2}{*}{$\begin{array}{c}\text { Antimicrobial } \\
\text { Agents }\end{array}$} & \multicolumn{2}{|c|}{$\operatorname{MIC}(\mu \mathrm{g} / \mathrm{mL})$} & \multirow{2}{*}{$\begin{array}{l}\text { \% susceptible } \\
\text { (breakpoint) }\end{array}$} \\
\hline & & $50 \%$ & $90 \%$ & \\
\hline \multicolumn{5}{|l|}{ S. pneumoniae } \\
\hline \multirow[t]{6}{*}{$(244)$} & Quinupristin/Dalfopristin & 0.5 & 1.0 & $100(\leq 1.0 \mu \mathrm{g} / \mathrm{mL})$ \\
\hline & Penicillin & 0.016 & 0.75 & $72(\leq 0.06 \mu \mathrm{g} / \mathrm{mL})$ \\
\hline & Cefotaxime & 0.032 & 0.5 & $90(\leq 0.5 \mu \mathrm{g} / \mathrm{mL})$ \\
\hline & Erythromycin & 0.064 & 0.75 & $88(\leq 0.25 \mu \mathrm{g} / \mathrm{mL})$ \\
\hline & Vancomycin & 0.25 & 0.5 & $100(\leq 1 \mu \mathrm{g} / \mathrm{mL})$ \\
\hline & Teicoplanin & 0.032 & 0.125 & $-{ }^{a}$ \\
\hline \multicolumn{5}{|l|}{ Other streptococci } \\
\hline \multirow[t]{6}{*}{$(82)$} & Quinupristin/Dalfopristin & 0.5 & 1.0 & $100(\leq 1.0 \mu \mathrm{g} / \mathrm{mL})$ \\
\hline & Penicillin & 0.016 & 0.38 & $87(\leq 0.12 \mu \mathrm{g} / \mathrm{mL})$ \\
\hline & Cefotaxime & 0.047 & 0.5 & $93(\leq 0.5 \mu \mathrm{g} / \mathrm{mL})$ \\
\hline & Erythromycin & 0.094 & 2.0 & $78(\leq 0.25 \mu \mathrm{g} / \mathrm{mL})$ \\
\hline & Vancomycin & 0.25 & 0.75 & $100(\leq 1 \mu \mathrm{g} / \mathrm{mL})$ \\
\hline & Teicoplanin & 0.06 & 0.25 & $-{ }^{a}$ \\
\hline
\end{tabular}

a. All pneumococci inhibited at $\leq 0.5 \mu \mathrm{g} / \mathrm{mL}$ and all streptococci inhibited at $\leq 2.0 \mu \mathrm{g} / \mathrm{mL}$.

\section{Figure 1.}

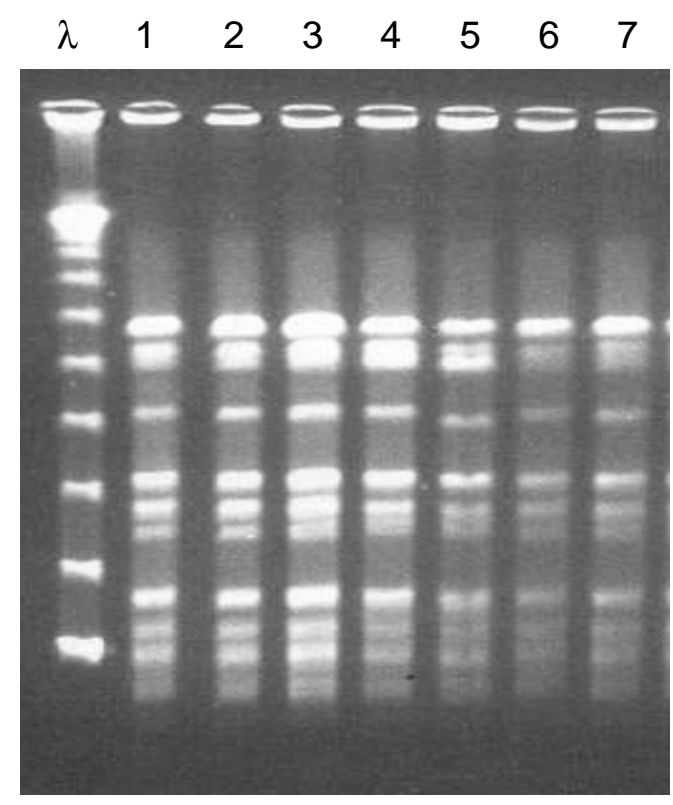


Table 2. Antimicrobial activity of quinupristin-dalfopristin and 11 other compounds tested against 747 S. aureus strains isolated in Latin America

\begin{tabular}{|c|c|c|c|c|c|}
\hline \multirow{2}{*}{$\begin{array}{l}\text { Organisms } \\
\text { (no. tested) }\end{array}$} & \multirow{2}{*}{$\begin{array}{l}\text { Antimicrobial } \\
\text { Agents }\end{array}$} & \multicolumn{2}{|c|}{$\mathrm{MIC}(\mathrm{mg} / \mathrm{mL})$} & \multicolumn{2}{|c|}{ \% susceptible/resistant (n) by: } \\
\hline & & $50 \%$ & $90 \%$ & Broth microdilution & Disk diffusion \\
\hline \multicolumn{6}{|l|}{ S. aureus } \\
\hline \multicolumn{2}{|l|}{ Oxacillin-susceptible } & & & $(n=178)$ & $(n=260)$ \\
\hline \multirow[t]{12}{*}{$(438)$} & $\begin{array}{l}\text { Quinupristin/ } \\
\text { Dalfopristin }^{\mathrm{a}}\end{array}$ & 0.25 & 0.25 & $100 / 0$ & $100 / 0$ \\
\hline & Erythromycin & 0.12 & $>16$ & $84 / 12$ & $76 / 14$ \\
\hline & Clindamycin & 0.12 & 0.25 & $97 / 2$ & $92 / 4$ \\
\hline & Ampicillin & 4 & $>16$ & $69 / 30$ & NT \\
\hline & Cefazolin & 0.25 & 0.5 & $100 / 0$ & $100 / 0$ \\
\hline & Cefotaxime & 0.5 & 1 & $100 / 0$ & $100 / 0$ \\
\hline & Vancomycin & 0.5 & 1 & $100 / 0$ & $100 / 0$ \\
\hline & Teicoplanin & 0.25 & 0.5 & $100 / 0$ & $100 / 0$ \\
\hline & Ciprofloxacin & 0.12 & 1 & $97 / 0$ & $92 / 2$ \\
\hline & Gentamicin & 0.25 & 2 & $93 / 0$ & NT \\
\hline & Chloramphenicol & 4 & 8 & $91 / 4$ & $94 / 4$ \\
\hline & Doxycycline & 2 & 4 & $96 / 0$ & $97 / 2$ \\
\hline \multirow{13}{*}{$\begin{array}{l}\text { Oxacillin-resistant } \\
(309)\end{array}$} & & & & $(n=144)$ & $(n=165)$ \\
\hline & $\begin{array}{l}\text { Quinupristin/ } \\
\text { Dalfopristin }^{\mathrm{a}}\end{array}$ & 0.25 & 0.5 & $100 / 0$ & $100 / 0$ \\
\hline & Erythromycin & $>16$ & $>16$ & $0 / 100$ & $8 / 91$ \\
\hline & Clindamycin & $>16$ & $>16$ & $5 / 95$ & $1 / 89$ \\
\hline & Ampicillin & $>16$ & $>16$ & $0 / 100$ & $0 / 100$ \\
\hline & Cefazolin & $>16$ & $>16$ & $0 / 100$ & $0 / 100$ \\
\hline & Cefotaxime & $>32$ & $>32$ & $0 / 100$ & $0 / 100$ \\
\hline & Vancomycin $^{\mathrm{b}}$ & 0.5 & 1 & $100 / 0$ & $100 / 0$ \\
\hline & Teicoplanin & 0.5 & 0.5 & $100 / 0$ & $97 / 0$ \\
\hline & Ciprofloxacin & 2 & 2 & $4 / 82$ & $13 / 84$ \\
\hline & Gentamicin & $>8$ & $>8$ & $4 / 82$ & $\mathrm{NT}$ \\
\hline & Chloramphenicol & $>16$ & $>16$ & $22 / 72$ & $54 / 44$ \\
\hline & Doxycycline & 8 & $>8$ & $40 / 10$ & $51 / 26$ \\
\hline
\end{tabular}

a Quinupristin/Dalfopristin susceptibility defined as MIC, $\leq 1 \mathrm{mg} / \mathrm{mL}$ or $\geq 19 \mathrm{~mm}[8,9]$.

b Only 1 strain with a MIC of $4.0 \mathrm{mg} / \mathrm{mL}$.

NT: Not tested. 
Table 3. Antimicrobial activity of quinupristin-dalfopristin and 11 other compounds tested against 446 coagulasenegative staphylococci (CoNS) strains isolated in Latin America

\begin{tabular}{|c|c|c|c|c|c|}
\hline \multirow{2}{*}{$\begin{array}{l}\text { Organisms } \\
\text { (no. tested) }\end{array}$} & \multirow{2}{*}{$\begin{array}{l}\text { Antimicrobial } \\
\text { Agents }\end{array}$} & \multicolumn{2}{|c|}{ MIC (mg/mL) } & \multicolumn{2}{|c|}{ \% susceptible/resistant (n) by: } \\
\hline & & $50 \%$ & $90 \%$ & Broth Microdilution & Disk Diffusion \\
\hline \multicolumn{6}{|l|}{ CoNS } \\
\hline \multirow{13}{*}{$\begin{array}{l}\text { Oxacillin-susceptible } \\
(171)^{\mathrm{a}}\end{array}$} & & & & $(n=52)$ & $(n=119)$ \\
\hline & $\begin{array}{l}\text { Quinupristin/ } \\
\text { Dalfopristin }\end{array}$ & 0.12 & 0.25 & $100 / 0$ & $100 / 0$ \\
\hline & Erythromycin & 0.12 & 16 & $69 / 25$ & 62 / 31 \\
\hline & Clindamycin & 0.12 & 0.5 & $92 / 4$ & $91 / 5$ \\
\hline & Ampicillin & 1 & 16 & $89 / 10$ & NT \\
\hline & Cefazolin & 0.25 & 0.5 & $100 / 0$ & $100 / 0$ \\
\hline & Cefotaxime & 0.5 & 1 & $100 / 0$ & $100 / 0$ \\
\hline & Vancomycin & 0.5 & 1 & $100 / 0$ & $100 / 0$ \\
\hline & Teicoplanin & 0.5 & 1 & $100 / 0$ & $100 / 0$ \\
\hline & Ciprofloxacin & 0.12 & 0.5 & $92 / 0$ & $91 / 6$ \\
\hline & Gentamicin & 0.12 & 0.5 & $94 / 4$ & NT \\
\hline & Chloramphenicol & 4 & 8 & $92 / 6$ & $94 / 5$ \\
\hline & Doxycycline & 2 & 8 & $89 / 2$ & $88 / 9$ \\
\hline \multirow{13}{*}{$\begin{array}{l}\text { Oxacillin-resistant } \\
(275)\end{array}$} & & & & $(n=102)$ & $(n=173)$ \\
\hline & $\begin{array}{l}\text { Quinupristin/ } \\
\text { Dalfopristin }\end{array}$ & 0.25 & 1 & $99 / 0^{c}$ & $100 / 0$ \\
\hline & Erythromycin & $>16$ & $>16$ & $27 / 72$ & $29 / 68$ \\
\hline & Clindamycin & $>16$ & $>16$ & $32 / 66$ & $42 / 52$ \\
\hline & Ampicillin & $>16$ & $>16$ & $0 / 100$ & NT \\
\hline & Cefazolin & $>16$ & $>16$ & $0 / 100$ & $0 / 100$ \\
\hline & Cefotaxime & $>32$ & $>32$ & $0 / 100$ & $0 / 100$ \\
\hline & Vancomycin ${ }^{\mathrm{d}}$ & 1 & 2 & $100 / 0$ & $100 / 0$ \\
\hline & Teicoplanin ${ }^{\mathrm{e}}$ & 1 & 2 & $100 / 0$ & $99 / 0$ \\
\hline & Ciprofloxacin & 2 & 2 & $28 / 58$ & $49 / 45$ \\
\hline & Gentamicin & 8 & $>8$ & $24 / 51$ & NT \\
\hline & Chloramphenicol & 16 & $>16$ & $37 / 51$ & $54 / 42$ \\
\hline & Doxycycline & 2 & 4 & $89 / 2$ & $66 / 27$ \\
\hline
\end{tabular}

a Oxacillin susceptibility was defined as $\mathrm{MIC} \leq 0.25 \mu \mathrm{g} / \mathrm{mL}$ or $\geq 18 \mathrm{~mm}[8,9]$.

$\mathrm{b}$ Quinupristin/dalfopristin susceptibility was defined as $\mathrm{MIC} \leq 1 \mu \mathrm{g} / \mathrm{mL}$ or $\geq 19 \mathrm{~mm}[8,9]$.

c One strain with a MIC of $2 \mu \mathrm{g} / \mathrm{mL}$.

$\mathrm{d}$ One strain with a MIC of $4 \mu \mathrm{g} / \mathrm{mL}$.

e One strain with a MIC of $8 \mu \mathrm{g} / \mathrm{mL}$ 
Table 4. Antimicrobial activity of quinupristin-dalfopristin and 6 other compounds tested against 429 enterococci strains isolated in Latin America

\begin{tabular}{|c|c|c|c|c|c|}
\hline \multirow{2}{*}{$\begin{array}{l}\text { Organisms } \\
\text { (no. tested) }\end{array}$} & \multirow{2}{*}{$\begin{array}{c}\text { Antimicrobial } \\
\text { Agents }\end{array}$} & \multicolumn{2}{|c|}{$\operatorname{MIC}(\mu \mathrm{g} / \mathrm{mL})$} & \multicolumn{2}{|c|}{$\%$ susceptible/resistant (n) by ${ }^{a}$} \\
\hline & & $50 \%$ & $90 \%$ & Broth Microdilution & Disk Diffusion \\
\hline \multicolumn{6}{|l|}{ E. faecium } \\
\hline \multirow{9}{*}{$\begin{array}{l}\text { Vancomycin- } \\
\text { susceptible (94) }\end{array}$} & & & & $(n=49)$ & $(n=45)$ \\
\hline & Quinupristin/ & 0.5 & 2 & $80 / 2$ & $96 / 2$ \\
\hline & Dalfopristin & & & & \\
\hline & Ampicillin & 0.5 & 16 & $84 / 16$ & $72 / 28$ \\
\hline & Vancomycin & 0.5 & 2 & $100 / 0$ & $100 / 0$ \\
\hline & Teicoplanin & 0.25 & 0.5 & $100 / 0$ & $100 / 0$ \\
\hline & Ciprofloxacin & 2 & $>2$ & $33 / 53$ & $24 / 24$ \\
\hline & Chloramphenicol & 4 & 16 & $88 / 8$ & $87 / 0$ \\
\hline & Doxycycline & 2 & $>8$ & $78 / 16$ & $61 / 22$ \\
\hline \multirow{9}{*}{$\begin{array}{l}\text { Vancomycin- } \\
\text { resistant (21) }\end{array}$} & & & & $(n=17)$ & $(n=4)$ \\
\hline & Quinupristin/ & 8 & 8 & $12 / 88$ & $100 / 0$ \\
\hline & Dalfopristin & & & & \\
\hline & Ampicillin & $>16$ & $>16$ & $0 / 100$ & $0 / 100$ \\
\hline & Vancomycin & $>16$ & $>16$ & $0 / 100$ & $0 / 100$ \\
\hline & Teicoplanin & $>16$ & $>16$ & $0 / 100$ & $0 / 100$ \\
\hline & Ciprofloxacin & $>2$ & $>2$ & $0 / 94$ & $0 / 50$ \\
\hline & Chloramphenicol & 8 & 8 & $94 / 6$ & $75 / 25$ \\
\hline & Doxycycline & 2 & 8 & $82 / 0$ & $75 / 25$ \\
\hline \multirow{9}{*}{$\begin{array}{l}\text { E. faecalis } \\
(223)\end{array}$} & & & & $(n=83)$ & $(n=140)$ \\
\hline & Quinupristin/ & 8 & $>16$ & $1 / 90$ & 13 / 71 \\
\hline & Dalfopristin & & & & \\
\hline & Ampicillin & 1 & 2 & $99 / 1$ & $93 / 7$ \\
\hline & Vancomycin & 1 & 2 & $98 / 2$ & $96 / 1$ \\
\hline & Teicoplanin & 0.12 & 0.12 & $98 / 2$ & $100 / 0$ \\
\hline & Ciprofloxacin & 2 & $>2$ & $44 / 32$ & $14 / 28$ \\
\hline & Chloramphenicol & 8 & $>16$ & $59 / 35$ & $69 / 14$ \\
\hline & Doxycycline & 4 & $>8$ & $60 / 23$ & $44 / 39$ \\
\hline \multirow{9}{*}{$\begin{array}{l}\text { Enterococcus } \\
\text { spp. (91) }\end{array}$} & & & & $(n=44)$ & $(n=47)$ \\
\hline & Quinupristin/ & 0.12 & 2 & $73 / 2$ & $94 / 4$ \\
\hline & Dalfopristin & & & & \\
\hline & Ampicillin & 0.50 & 2 & $91 / 9$ & $89 / 11$ \\
\hline & Vancomycin & 0.25 & 4 & $91 / 9$ & $87 / 0$ \\
\hline & Teicoplanin & 0.12 & 0.25 & $91 / 9$ & $94 / 2$ \\
\hline & Ciprofloxacin & 1 & $>2$ & $72 / 14$ & $11 / 43$ \\
\hline & Chloramphenicol & 4 & 8 & $91 / 9$ & $36 / 30$ \\
\hline & Doxycycline & 2 & 2 & $98 / 2$ & $23 / 60$ \\
\hline
\end{tabular}

a Quinupristin/dalfopristin susceptibility was defined as MIC, $\leq 1 \mu \mathrm{g} / \mathrm{mL}$ or $\geq 19 \mathrm{~mm}[8,9]$. 
Table 3 shows the results for the CoNS species (446 strains). Like the results for $S$. aureus listed above, quinupristin/dalfopristin, vancomycin, and teicoplanin were the most active compounds with quinupristin/ dalfopristin usually being 2- to 4-fold more potent. Similar to ORSA strains, oxacillin-resistant CoNS showed high rates of resistance to all other tested antimicrobial agents and only doxycycline inhibited more than one-half of those strains. On the other hand, oxacillin-susceptible CoNS showed high rates of susceptibility with more than $90 \%$ of strains being inhibited by most antimicrobial agents, except erythromycin (62\% to $69 \%$ susceptibility) and doxycycline (88\% to $89 \%$ susceptibility). Ciprofloxacin inhibited $91 \%$ to $92 \%$ of the oxacillin-susceptible CoNS strains at the susceptible breakpoint $(\leq 1 \mu \mathrm{g} / \mathrm{mL})$, but only $28 \%$ to $49 \%$ of the oxacillin-resistant strains were ciprofloxacin-susceptible. One oxacillin-resistant CoNS isolate with intermediate resistance to quinupristin/dalfopristin (MIC, $2 \mu \mathrm{g} / \mathrm{mL}$ ), and 1 isolate with intermediate resistance to teicoplanin (MIC, $8 \mu \mathrm{g}$ / $\mathrm{mL}$ ) were detected.

In Table 4, only the most active drugs tested against the enterococci (429 strains) are listed. Among those isolates, $338(78.8 \%)$ were identified to the species level by the participating laboratories, dominated by E. faecalis and E. faecium. E. faecium isolates were divided according to their susceptibility to vancomycin. Among the 115 E. faecium isolates evaluated, 21 (18.2\%) were found to be vancomycin-resistant. Quinupristin/dalfopristin showed good activity against vancomycin-susceptible E. faecium ( $\mathrm{MIC}_{90}, 2 \mu \mathrm{g} / \mathrm{mL}$ ) with only 2 isolates ( $2 \%$ ) showing resistance. However, $18 \%$ of the vancomycin-susceptible isolates evaluated by broth microdilution (Brazil), and $2 \%$ of the isolates tested by the disk diffusion method demonstrated intermediate resistance (MIC, $2 \mu \mathrm{g} / \mathrm{mL}$ or zone diameter of $16 \mathrm{~mm}$ to $18 \mathrm{~mm}$ ) to quinupristin/ dalfopristin.

Of the 21 vancomycin-resistant $E$. faecium isolates, 17 were from Brazil and 4 from Argentina. All the Brazilian vancomycin-resistant strains were isolated in São Paulo (3 hospitals), and 15 of those strains (88\%) were also resistant to quinupristin/dalfopristin. Those
15 E. faecium strains that showed resistance to both vancomycin and quinupristin/dalfopristin, and 2 vancomycin-resistant quinupristin/dalfopristinsusceptible E. faecium strains from Argentina were typed by PFGE. Thirteen of the 15 Brazilian isolates $(87 \%$ ) presented a unique major PGFE pattern (called A), indicating clonal dissemination (Figure 1). The Argentinean strains showed PFGE patterns different from each other and from the epidemic Brazilian clone. Ampicillin was active against $72 \%$ to $84 \%$ of the vancomycin-susceptible $E$. faecium, but none of the vancomycin-resistant strains were ampicillinsusceptible. On the other hand, chloramphenicol and doxycycline showed moderate in vitro activity against both vancomycin-susceptible and -resistant $E$. faecium. Ampicillin, vancomycin, and teicoplanin were very active against $E$. faecalis and Enterococcus spp. (Table 4). As expected, quinupristin/dalfopristin showed limited activity against $E$. faecalis and variable activity against Enterococcus spp., since these strains were not identified to the species level and could contain some isolates of E. faecium.

\section{Discussion}

Antimicrobial resistance has developed very rapidly in recent years, especially among Gram-positive cocci [12-14]. This has created an acute need for effective agents. Quinupristin/dalfopristin (formerly RP 59500) seems to be a potential alternative therapeutic antimicrobial for use in institutions facing the problem of multiresistant Gram-positive cocci. This compound is a water-soluble synthetic streptogramin combination of quinupristin (a streptogramin B) and dalfopristin (a streptogramin A) at a ratio of 30:70 [7]. Its described activity is focused against Gram-positive species and a few Gram-negative organisms. The action of streptogramins is generally bactericidal, in contrast to either component alone or structurally similar macrolide antimicrobials. Quinupristin/dalfopristin activity is 10fold to 100-fold greater than its component potencies and extends to organisms with documented MLS $_{B}$ resistance patterns $[15,16]$. Resistance to 
streptogramin combinations can occur by one of several resistance mechanisms including modified target, active efflux, or block of permeases, but these are generally uncommon [15-17].

In this multicenter, international investigation, quinupristin/dalfopristin demonstrated a broad antimicrobial spectrum and potency against $S$. pneumoniae (100\% susceptibility), Streptococcus spp. (100\% susceptibility), S. aureus (100\% susceptibility), CoNS (100\% susceptibility), and vancomycinsusceptible E. faecium ( $80 \%$ to $96 \%$ susceptibility). The streptogramin activity was evaluated in a background of increasing clinical resistance. The resistance of S. pneumoniae to penicillin and macrolide is already very high in some Latin American countries, and seems to be continuously increasing $[18,19]$. Staphylococci resistant to oxacillin and many other antimicrobial agents are also very frequent in Latin American countries $[20,21]$. In addition, we have recently been confronted with the problem of vancomycin-resistant enterococci (VRE) [22].

The compromised activity of quinupristin/dalfopristin against some strains of vancomycin-resistant $E$. faecium detected in this study was unexpected because in a large, multicenter study performed in 1996 to1997 in the U.S. (including 326 vancomycin-resistant $E$. faecium strains), the $\mathrm{MIC}_{50}$ and $\mathrm{MIC}_{90}$ was $0.5 \mu \mathrm{g}$ / $\mathrm{mL}$ and $1 \mu \mathrm{g} / \mathrm{mL}$, respectively [7]). This unusual resistance pattern was mainly due to the intra- and interhospital dissemination of a single resistant clone. The results of molecular typing were very important to understand the rapid emergence of VRE in Brazil. Until recently, this pathogen was rarely isolated in Brazilian hospitals [22]. This epidemic increase of VRE prevalence is mainly due to the dissemination of various resistant clones. Unfortunately, the predominant Brazilian VRE clone has also acquired low-level resistance to quinupristin/dalfopristin.

The bacteriostatic effects of quinupristin/dalfopristin on $E$. faecium, especially those isolates with resistance to vancomycin and macrolides, must be emphasized [23]. Streptogramins would be expected to kill only those enterococcal isolates with erythromycin susceptible test results [23]. Substantial clinical (73.6\%) and microbiological (70.5\%) success rates have been obtained by treatment with quinupristin/dalfopristin for vancomycin-resistant E. faecium infections in patients with a severe underlying condition such as diabetes mellitus, organ transplantation, mechanical ventilation, dialysis, chronic liver disease with cirrhosis, or oncological disorder, for which no other therapeutic alternative has been available [24]. However, physicians who wish to use quinupristin/dalfopristin for a bactericidal action, especially in clinical settings such as endocarditis, should seek additional erythromycin test results to guide therapy within appropriate indications [25]. Such attention to detail in the microbiology diagnosis and susceptibility tests should limit the occurrence of resistance emerging to quinupristin/dalfopristin chemotherapy [26].

The microbiology laboratory diagnosis will have a unique, but correctable, problem with the quinupristin/ dalfopristin susceptibility tests. The selective potency of quinupristin/dalfopristin against E. faecium among all other Enterococcus species has uncovered the potential for false resistance due to misidentification of enterococcal organisms by several commercial systems $[27,28]$. A correct species identification is essential for the appropriate clinical use of this new compound. Supplemental rapid tests (motility, pigment production, xylose and/or arabinose fermentation) and the examination of antimicrobial susceptibility patterns (ampicillin resistance usually indicates E. faecium; ampicillin-susceptibility favors an E. faecalis identification) could minimize the reporting of inaccurate results [29].

In conclusion, after testing nearly 2,000 clinical bacterial isolates from 15 Latin American medical centers, the results presented here indicate the favorable spectrum of quinupristin/dalfopristin compared to other contemporary antimicrobial agents. This streptogramin (quinupristin/dalfopristin), along with other investigational agents [12, 14, 30-32], is urgently needed for therapy of serious Gram-positive infections and offers an alternative to glycopeptide therapy. The role of quinupristin/dalfopristin in infectious disease practice still awaits more extensive clinical data and experience. This agent should be selected based on 
the treatment benefit for the patients at risk versus the potential for adverse drugs events. Our results also demonstrate the importance of antimicrobial resistance surveillance programs in guiding empirical regimens, and for focusing intervention controls of antimicrobial resistance in distinct geographic areas (example: São Paulo), hospitals, and possibly within services/wards.

\section{Acknowledgements}

The authors wish to thank A. C. Gales and L. Sejas for their significant contributions to this investigation. This study was funded by a research grant from RhonePoulenc Rorer. The GSMART Latin America Study Group is composed by:H. S. Sader (Brazil-Latin America Coordinator); J. Sampaio (Lâmina Laboratory, Rio de Janeiro, Brazil); C. Zoccoli (Santa Luzia Laboratory, Florianópolis, Brazil); C. Mendes (LIM-54, Infectious Diseases Department, HC-FMUSP, São Paulo, Brazil); C. G. Dias (Moinho de Vento Hospital, Porto Alegre, Brazil); L. Mimiça (Santa Casa da Misericórdia de São Paulo, São Paulo, Brazil); J. Samyevsky (Microbiology Laboratory C.E.M.I.C., Buenos Aires, Argentina); J. M. Casellas (San Lucas Hospital, Martinez, Argentina); A. Monterisi (Hospital Nacional de Clínicas, Cordoba, Argentina); M.D. Sparo (Hospital Ramón Santamaria, Tandil, Argentina); R. Notario(Instituto de Doagnostico, Rosário, Argentina); V. Prado (Faculdad de Medicina de Chile, Santiago, Chile); E. Palaveccino (Universidad Catolica del Chile, Santiago, Chile); J. Zurita (Hospital Vozandes, Quito, Ecuador); M. Guzmán-Blanco (San Bernardino, Caracas, Venezuela).

\section{References}

1. Pfaller M.A., Jones R.N., Doern G.V., et al. Bacterial pathogens isolated from patients with bloodstream infection: Frequencies of occurrence and antimicrobial susceptibility patterns from the SENTRY antimicrobial surveillance program (United States and Canada, 1997). Antimicrob Agents Chemother 1998; 42: 1762-70.
2. Pfaller M.A., Jones R.N., Doern G.V., et al. Survey of bloodstream infections attributable to Gram-positive cocci: Frequency of occurrence and antimicrobial susceptibility of isolates collected in 1997, in the United States, Canada, and Latin America, from the SENTRY Antimicrobial Surveillance Program. Diagn Microbiol Infect Dis 1999;33:283-97.

3. Sader H.S., Sampaio J.L.M., Zoccoli C., Jones R.N. Results of the SENTRY antimicrobial surveillance program results in three Brazilian medical centers for 1997. Braz J Infect Dis 1999;3:63-79.

4. Sader H.S., Jones R.N., Gales A.C., et al. Antimicrobial susceptibility patterns for pathogens isolated from patients in Latin American medical centers with a diagnosis of pneumonia: Results from the SENTRY Antimicrobial Surveillance Program (1997). Diagn Microbiol Infect Dis 1998;32:289-301.

5. Jones M.E., Sader H.S., Verhoef J., et al. Current state of susceptibility to glycopeptides in Staphylococcus species from an international resistance surveillance program. [Letter]. J Antimicrob Chemother 1998;42:119-21.

6. Del'Alamo L., Cereda R.F., Tosin I., et al. Antimicrobial susceptibility of coagulase-negative staphylococci and characterization of isolates with decreased susceptibility to glycopeptides. Diagn Microbiol Infect Dis 1999;34:185-91.

7. Jones R.N., Ballow C.H., Biedenbach D.J., et al. Antimicrobial activity of quinupristin-dalfopristin (RP 59500, Synercid) tested against over 28,000 recent clinical isolates from 200 medical centers in the United States and Canada. Diagn Microbiol Infect Dis 1998;31:437-51.

8. National Committee for Clinical Laboratory Standards. Methods for Dilution Antimicrobial Susceptibility Tests for Bacteria that Grow Aerobically, 5th ed.: Approved Standard, M7-A5. Wayne, PA, 2000.

9. National Committee for Clinical Laboratory Standard. Performance Standards for Antimicrobial Susceptibility Testing; $10^{\text {Th }}$ informational supplement M100-S10. Wayne, PA, 2000.

10. National Committee for Clinical Laboratory Standard. Performance standards for antimicrobial susceptibility testing; $8^{\text {Th }}$ informational supplement M100-S8. Wayne, PA, 1998.

11. Pfaller M.A., Sader H.S., Hollis R.J. Chromosomal restriction fragment analysis by pulsed-field gel electrophoresis. (Ed) Isenberg HD. Clinical Microbiology Procedures Handbook. 1992;Suppl 1:10.5c.1-12.

12. Cormican M.G., Jones R.N. Emerging resistance to antimicrobial agents in Gram-positive bacteria. Drugs 1996;51(suppl 1):6-12. 
13. Hiramotsu K., Hanaki H., Ino T., et al. Methicillin-resistant Staphylococcus aureus clinical strains with reduced vancomycin susceptibility. J Antimicrob Chemother 1997;40:135-46.

14. Landman D., Quale J.M. Management of infections due to resistant enterococci: A review of therapeutic options. J Antimicrob Chemother 1997;40:161-70.

15. Pechere J.C. Streptogramins: A unique class of antibiotics. Drugs 1996;51(suppl 1):13-9.

16. Vannuffel P., Cocito C. Mechanism of action of streptogramins and macrolides. Drugs 1996;51(suppl 1):20-30..

17. Rende-Fournier R., LeClerq R., Galimand M., et al. Identification of the satA gene encoding a streptogramin A acetyltransferase in Enterococcus faecium BM4145. Antimicrob Agents Chemother 1993;39:139-43.

18. Tomasz A., Corso A., Members of the PAHO/ Rockefeller University Workshop, et al. Molecular epidemiologic characterization of penicillin-resistant Streptococcus pneumoniae recovered in six Latin American countries: An overview. Microbial Drug Resist 1998; 4: 195-207.

19. Sader H.S., Gales A.C., Granacher T.L., et al. Prevalence of antimicrobial resistance among respiratory tract isolates in Latin America: Results from SENTRY Antimicrobial Surveillance Program (1997-98). Braz J Infect Dis 2000;4:246-55.

20. Sader H.S., Jones R.N., Gales A.C., et al. Antimicrobial susceptibility of patterns for pathogens isolated from patients in Latin American medical centers with a diagnosis of pneumonia: Results from the SENTRY Antimicrobial Surveillance Program (1997). Diagn Microbiol Infect Dis 1998;32:289-301.

21. Sader H.S., Pfaller M.A., Jones R.N., et al. Bacterial pathogens isolated from patients with bloodstream infections in Latin America, 1997: Frequency of occurrence and antimicrobial susceptibility patterns from the SENTRY Antimicrobial Surveillance Program. Braz J Infect Dis 1999;3:97-110.

22. Dalla Costa L.M., Souza D.C., Martins L.T.F., et al. Vancomycin-resistant Enterococcus faecium: First case in Brazil. Braz J Infect Dis 1998; 2:160-3.

23. Caron F., Gold H.S., Wennersten C.B., et al. Influence of erythromycin resistance, inoculum growth phase, and incubation time on assessment of the bactericidal activityof RP 59500 (quinupristin/dalfopristin) against vancomycin-resistant Enterococcus faecium. Antimicrob Agents Chemother 1997;41:2749-53.

24. Moellering R.C., Linden P.K., Reinhardt J., et al. The efficacy and safety of quinupristin/dalfopristin for the treatment of infections caused by vancomycin-resistant Enterococcus faecium. Antimicrob Agents Chemother 1999;44:251-61.
25. Linden P.K., Pasculle A.W., Manez R., et al. Differences in outcomes for patients with bacteremia due to vancomycin-resistant Enterococcus faecium or vancomycin-susceptible E. faecium. Clin Infect Dis 1996;22:663-70.

26. Chow J.W., Donabedian S.M., Zervos M.J. Emergence of increased resistance to quinupristin/dalfopristin during therapy Enterococcus faecium bacteremia. Clin Infect Dis 1997;24:90-1.

27. Jones R.N., Marshall S.A., Pfaller M.A., et al. Nosocomial enterococcal bloodstream infections in the SCOPE Program: antimicrobial resistance, species occurrence, molecular testing results, and laboratory testing accuracy. SCOPE Hospital Study Group. Diagn Microbiol Infect Dis 1997;29:95-102.

28. Sader H.S., Biedenbach D., Jones R.N. Evaluation of Vitek and API 20S for species identification of enterococci in comparison with a conventional test scheme. Diagn Microbiol Infect Dis 1995;22:315-9.

29. Willey B.M., Jones R.N., McGeer A., et al. Practical approach to the identification of clinically relevant Enterococcus species. Diagn Microbiol Infect Dis 1999;34:165-71.

30. Jones R.N., Marshall S.A., Erwin M.E. Antimicrobial activity and spectrum of SCH 27899 (Ziracin) tested against Gram-positive species including recommendations for routine susceptibility testing methods and quality control. Quality Control Study Group. Diagn Microbiol Infect Dis 1999;34:103-10.

31. Jones R.N., Low D.E., Pfaller M.A. Epidemiologic trends in nosocomial and community-acquired infections due to antibiotic-resistant Gram-positive bacteria: the role of streptogramins and other newer compounds. Diagn Microbiol Infect Dis 1999;33:101-12.

32. Diekema D.I., Jones R.N. Oxazolidinones: a review. Drugs 2000;59:7-16. 